\title{
Modelling and validation of near-field Diesel spray CFD simulations based on the $\Sigma-Y$ model
}

\author{
J.M. Desantes ${ }^{1}$, J.M. García-Oliver ${ }^{1}$, J.M. Pastor¹, A. Pandal ${ }^{2}$, B. Naud ${ }^{3}$, K. Matusik ${ }^{4}$, D. \\ Duke $^{4}$, A. Kastengren ${ }^{4}$, C. Powell ${ }^{4}$, D.P. Schmidt ${ }^{5}$ \\ ${ }^{1} \mathrm{CMT}$ Motores Térmicos, Universitat Politècnica de València, Spain \\ 2 Área de Mecánica de Fluidos - Dpto. Energía, Universidad de Oviedo, Spain \\ ${ }^{3}$ CIEMAT, Spain \\ ${ }^{4}$ Argonne National Laboratory, USA \\ ${ }^{5}$ University of Massachusetts, Amherst, USA \\ *Corresponding author: jopasen@mot.upv.es
}

\begin{abstract}
Diesel spray modelling still remains a challenge, especially in the dense near-nozzle region. This region is difficult to experimentally access and also to model due to the complex and rapid liquid and gas interaction. Modelling approaches based on Lagrangian particle tracking have struggled in this area, while Eulerian modelling has proven particularly useful. An interesting approach is the single-fluid diffuse interface model known as $\Sigma-Y$, based on scale separation assumptions at high Reynolds and Weber numbers. Liquid dispersion is modelled as turbulent mixing of a variable density flow. The concept of surface area density is used for representing liquid structures, regardless of the complexity of the interface.

In this work, an implementation of the $\Sigma-Y$ model in the OpenFOAM CFD library is applied to simulate the ECN Spray $A$ in the near nozzle region, using both RANS and LES turbulence modelling. Assessment is performed with measurements conducted at the Advanced Photon Source at Argonne National Laboratory (ANL). The ultra-smallangle x-ray scattering (USAXS) technique has been used to measure the interfacial surface area, and x-ray radiography to measure the fuel dispersion, allowing a direct evaluation of the $\Sigma-Y$ model predictions.
\end{abstract}

\section{Keywords}

Sprays, Diesel, atomization, CFD, OpenFOAM, X-ray

\section{Introduction}

Fuel injection and subsequent spray development are critical factors for charge preparation, combustion development and pollutants formation in engines. The liquid atomization process occurs at extremely small length scales and high speeds in current injection systems, which complicates both the investigation and modelling of spray flow, especially in the near-nozzle region. The lack of optical accessibility, except by means of special diagnostic techniques [1][2], hinders the flow characterization and the development of predictive primary atomization models.

The common spray modelling approaches, based on the representation of the liquid phase using a Lagrangian framework [3], are not well suited to represent this dense region, while fully Eulerian approaches have recently shown their potential to simulate near-nozzle physics [4][5]. Complex modelling techniques devoted to capturing the liquid-gas interface [6][7][8] have been successfully applied to simulate initial spray development, but the computational requirements can make those calculations impractical for spray applications in combustion systems due to high Reynold and Weber numbers.

Under these conditions, one may assume a separation of the large scale flow features, such as mass transport, from the atomization process occurring at smaller scales, as proposed in[9][10]. Then large scale liquid dispersion can be modelled as the turbulent mixing of a variable density fluid. For atomization, the surface density concept is introduced in order to evaluate the mean size of liquid fragments, assuming that interfacial details are smaller than the mesh size. The end result is a diffuse-interface treatment in an Eulerian framework. This framework is naturally extensible to near critical or super-critical regimes [11]. Rather than directly tracking the unresolved interface features, they are modelled in an Eulerian framework.

These diffuse-interface Eulerian spray models have two common elements: a model for the transport of liquid (or gas) and a model for the evolution of the interfacial surface area. The density of interfacial area is typically denoted by Sigma $(\Sigma)$ while the liquid fraction is denoted by $\mathrm{Y}$. Hence, we refer to the strictly Eulerian model as a $\Sigma-Y$ approach, in contrast to ELSA (Eulerian-Lagrangian Spray Atomization), which includes a transition to Lagrangian particle tracking [12][13]. 
The transport of the liquid employs mass-averaged convection along with turbulent mixing. This model is derived from basic Favre averaging or LES filtering [14]. Thus, the accuracy of the liquid fraction transport is largely dependent on the accuracy of the two-phase turbulent modelling. Despite the challenges of such modelling, there is at least an extensive theoretical basis to deal with the unclosed terms, putting the $Y$ transport equation on a much firmer foundation than the interfacial evolution.

However, the model for the interface evolution is somewhat more speculative, with several unclosed terms [15]. There are several interface modelling approaches that have been applied to sprays as researchers have explored competing ideas of how these terms should be treated [10][7][15].

In this paper, the $\Sigma-Y$ model is evaluated in diesel sprays near-nozzle region by comparing its predictions with experiments conducted at Argonne National Laboratory (ANL) within the Engine Combustion Network (ECN) framework [16]. Those experiments provide unique data using x-ray radiography [1] and the recently developed ultra-small-angle x-ray scattering (USAXS) [17].

Previous works [4][18][19] have shown liquid spray dispersion predictions compared to x-ray radiography data, and the noticeable effect of the turbulence model in those RANS based simulations. In this paper, a LES approach is also applied and its impact on spray dispersion is evaluated.

The interfacial density predictions have rarely been validated, and these validations have been in the context of downstream drop size [20][21], which is not ideal since we wish to avoid the assumption that the liquid is in the form of droplets. A few prior examples used DNS simulations [7] for validation, and in this paper the validation is performed via USAXS measurements, which directly measures the interfacial surface density. In this work, the formulation proposed by [22] has been assessed and compared with recent modelling results [23].

In summary, we wish to validate the transport of liquid mass and assess when the model for interfacial evolution is accurate.

\section{Experimental methodology}

Two different techniques, developed and performed at the Advanced Photon Source at ANL, have been applied in order to characterize diesel spray structure: X-ray radiography and Ultra-small angle x-ray scattering (USAXS)

The $x$-ray radiography [24] experiments provide a path-length-integrated measure of the fuel density along one beam path through the spray. To measure the spatial distribution of the fuel, a two-dimensional raster-scan approach is used, with each point measured from a different set of spray events. To further improve the signal/noise ratio in the final data, each data point is an average of 128-256 individual spray events. As such, the final data represent the ensemble averaged three-dimensional fuel density projected onto a plane. The fuel distribution data are thus reported as a Projected Mass Density (PMD), providing valuable information concerning liquid spray dispersion.

The USASX technique was used in order to evaluate interfacial surface area [17], due to its ability to interrogate the dense region and provide quantitative information about the complex interface without resorting to the assumption that the liquid is in the form of droplets. In performing these experiments, the Bonse-Hart instrument at the 9-IDbeamline measures the scattering intensity, Iscat $(q)$, for a wide range of scattering vectors, $q$ [25]. A beam of $\mathrm{x}$-rays at $17.9 \mathrm{keV}$ is first shaped into a $100 \times 500 \mu \mathrm{m} \mathrm{H} \times \mathrm{V}$ spot with a set of high precision $2 \mathrm{D}$ slits. The incoming beam is collimated with a pair of $\mathrm{Si}(220)$ crystals before impinging on the spray, from which the incident x-rays scatter at small angles. The scattered x-rays are filtered downstream with a pair of $\mathrm{Si}(220)$ analyzer crystals, and the scattered photons are measured with a detector. The pair of analyzer crystals is rotated to measure the transmitted beam intensity as a function of scattering vector. The scattering vector was varied between $1 \times 10^{-4} \AA^{-}$ ${ }^{1}<q<1 \times 10^{-2} \AA^{-1}$ with a step size of $1 \times 10^{-5} \AA^{-1}$ at low $q$, with increasing step size for larger $q$. The scattering intensity as a function of $q$ was measured at axial distances ranging from 1 to $20 \mathrm{~mm}$ downstream of the injection nozzle, at the centerline of the spray. Once $I_{\text {scat }}(q)$ is known, the differential cross-section may be calculated, and related to the total shape and surface area per volume of fuel droplets, with post-processing performed using the Irena data analysis package [26].

A solenoid diesel injection nozzle provided by the Engine Combustion Network (ECN); the single-hole Spray A 210675 nozzle, with a nominal hole diameter of $90 \mu \mathrm{m}$, was investigated. The injector was mounted horizontally in a $0.5 \mathrm{~L}$ vessel pressurized with $\mathrm{N} 2$ gas, which also supplied a purge flow of approximately 4 standard L/min in order to inhibit droplet accumulation within the domain during measurements.

In order to provide useful data for comparison with other ECN experiments, the experimental conditions have been matched as closely as possible to the ECN Spray A specification [16]. The specified injector, fuel, rail and injections line were used, but ambient gas is at room temperature instead of the prescribed high-temperature $(900 \mathrm{~K})$. The current experiments have, however, matched the ambient density of the Spray A specification, at the expense of not matching the ambient pressure; density is expected to be a more critical parameter in fuel-air mixing than 
pressure [27]. In addition, two additional injection pressures and one ambient pressure were also consider for the USAXS experiments, as shown in Table 1.

Table 1. Operating conditions in experiments

\begin{tabular}{|l|c|c|c|c|}
\hline Injector & \multicolumn{2}{|c|}{ Rail Pressure (bar) } & Chamber Pressure (bar) \\
\hline Spray A 210675 & \multicolumn{2}{|c|}{1500} & 6.7 \\
\hline Spray A 210675 & 1500 & 1000 & 500 & 20 \\
\hline
\end{tabular}

\section{Modelling approach}

To track the dispersion of the liquid phase an indicator function is used, taking a value of unity in the liquid phase and zero in the gas phase. The liquid volume fraction is denoted as $\bar{Y}$, and the mass-averaged fraction is defined as $\widetilde{\boldsymbol{Y}}=\overline{\boldsymbol{\rho} Y} / \overline{\boldsymbol{\rho}}$. Favre averaging or filtering the transport equation for the liquid mass fraction yields Eq. (1).

$$
\frac{\partial \bar{\rho} \widetilde{Y}}{\partial t}+\frac{\partial \bar{\rho} \widetilde{u_{\imath}} \widetilde{Y}}{\partial x_{i}}=-\frac{\partial \tau_{y i}}{\partial x_{i}}
$$

The turbulent diffusion liquid flux term $\boldsymbol{\tau}_{\boldsymbol{y} i}$, captures the effect of the relative velocity between the two phases [14]. This term is modelled using a standard turbulent gradient flux model, which successfully worked for Diesel spray compared to DNS results [15].

Under the assumption that the two phases form an immiscible mixture, the mass-averaged value of the indicator function is related to the density by Eq. (2). An equation of state is then assigned to each phase. The gas phase obeys an ideal gas law, that the liquid phase maintains a constant linear compressibility, and that both phases experience the same pressure.

$$
\frac{1}{\bar{\rho}}=\frac{\widetilde{Y}}{\rho_{l}}+\frac{1-\widetilde{Y}}{\rho_{g}}
$$

To close the above system of equations, the temperature is obtained from a bulk mixture enthalpy equation, where $h_{l}$ and $h_{g}$ denote the enthalpy of the liquid and gas phases respectively:

$$
h(T)=\widetilde{Y} \cdot h_{l}(T)+(1-\widetilde{Y}) \cdot h_{g}(T)
$$

The solution of the above equations fully characterizes the large-scale bulk motion of the flow. Conversely, the small scale atomization is modelled by solving a transport equation for the evolution of the interphase surface area density, Sigma ( $\Sigma$ ). This surface density is based on Vallet and Borghi [9] formulation, which includes source terms to account for generation due to the growth of fluid instabilities (i.e. Kelvin-Helmholtz) and the destruction of surface due to droplet coalescence (in the case of dispersed flow). However, the most common form for the combination of these two source terms is the restoration to an equilibrium value $\left(\bar{\Sigma}_{e q}\right)$ or critical surface density to which the local surface density is driven [7][21]:

$$
\frac{\partial \widetilde{\Sigma}}{\partial t}+\frac{\partial \widetilde{u_{j}} \widetilde{\Sigma}}{\partial x_{j}}-\frac{\partial}{\partial x_{j}}\left(D_{\Sigma} \frac{\partial \widetilde{\Sigma}}{\partial x_{j}}\right)-C_{\Sigma} \widetilde{\Sigma}\left(1-\frac{\widetilde{\Sigma}}{\bar{\Sigma}_{e q}}\right)-S_{\Sigma_{i n i t}}=0
$$

The $\bar{\Sigma}_{e q}$, already mentioned, is computed from an equilibrium Weber number [22], instead of using an equilibrium droplet radius, as in a recent work [23], in order to avoid any kind of assumption of spherical droplets.

$$
\bar{\Sigma}_{e q}=\alpha_{2} \frac{\left(\rho_{l}+\rho_{g}\right) \bar{Y}(1-\bar{Y}) \widetilde{k}}{\sigma}
$$

Then, the coefficient $\boldsymbol{C}_{\boldsymbol{\Sigma}}$ is modeled as the inverse of the turbulent time scale:

$$
C_{\Sigma}=\alpha_{1} \frac{\tilde{\varepsilon}}{\widetilde{k}}
$$

Note the presence of the two modelling constants $\left(\boldsymbol{\alpha}_{1}, \boldsymbol{\alpha}_{2}\right)$, by default equal to 1.0 [15][22], which should be calibrated.

Finally, as can be seen all the source terms that are involved in this equation are proportional to the interface surface density. As a result, there will be no production if there is no interface. Therefore, a proper initialization should be made by means of the term $\boldsymbol{S}_{\boldsymbol{\Sigma}_{\text {init }}}$. For that purpose, another update is presented in this work with respect to [23] in order to prevent any possible grid dependency. In a similar way as in [7], the initialization corresponds to a production due to the liquid/gas mixing process: 


$$
\begin{gathered}
\Sigma_{\text {init }}=2 \bar{\rho} \frac{v_{\mathrm{t}}}{S c} \frac{6 \bar{\rho}}{\rho_{l} \rho_{g} l_{t}} \frac{\partial \widetilde{Y}}{\partial x} \frac{\partial \widetilde{Y}}{\partial x}, \text { if } \widetilde{Y}(1-\widetilde{Y}) \leq 0.001 \\
\Sigma_{\text {init }}=2 \frac{v_{\mathrm{t}}}{S c} \frac{\widetilde{\Sigma}}{\widetilde{Y}(1-\widetilde{Y})} \frac{\partial \widetilde{Y}}{\partial x} \frac{\partial \widetilde{Y}}{\partial x}, \text { otherwise }
\end{gathered}
$$

The previously described model equations have been implemented into a solver [20] constructed by using the OpenFOAM [28] CFD libraries.

\section{Computational model set-up}

The calculations have been performed for the ECN Spray A using a 3-D computational domain with $80 \mathrm{~mm}$ length and $25 \mathrm{~mm}$ in radius. The mesh is structured with non-uniform grid resolution. There are 20 cells along the orifice diameter, keeping an aspect ratio close to one in the near nozzle region and is stretched in axial and radial directions. This mesh construction is the result of different sensitivity studies previously performed, including an evaluation of nozzle resolution [29].

The boundaries included non-slip conditions for the walls and non-reflective conditions for the open-ends. The inlet boundary condition uses the measured mass-flow rate in order to get the bulk injection velocity. LES cases use a specific inlet condition in order to generate turbulent fluctuations with a given statistical profile, based on the proposals by [30][31] and following the method described in [32]. The characteristic correlation length scale is twice the orifice grid resolution in order to resolve the generated eddies. The initial fluctuation level can be imposed over the mean velocity profile. This inlet profile is then rescaled in order to obtain the prescribed bulk injection velocity at each time-step.

The turbulent flow field for RANS is solved by a standard $k-\varepsilon$ model with a round-jet corrected value (1.6) for the $\mathrm{C}_{1 \varepsilon}$ constant [33], which provided good results for spray near [4] and far-field [20]. An eddy-viscosity based sub-grid model, derived from the analysis of the singular values of the resolved velocity gradient tensor [34], is used for LES cases. Time derivative terms are solved by a first order Euler scheme for RANS cases while LES uses a secondorder backward scheme. Divergence terms are solved by a Gamma NVD scheme for both turbulence modelling.

\section{Liquid spray dispersion: Projected mass density results}

Previous work has shown the $\Sigma$-Y RANS predictions compared to X-ray data [18][29]. Figure 1 shows projected mass density (PMD) at different axial locations downstream the nozzle. Note that the experimental profiles have been shifted to be centred at the axis in order to avoid the effect produced by the offset of the orifice outlet with respect to the needle axis in this injector (Serial\# 210675). It is shown in this figure that the $\Sigma-Y$ model is able to predict the shape of the PMD distributions in the near-nozzle region. The model captures the measured PMD data both in peak and radial dispersion at 0.1 and $2 \mathrm{~mm}$ sections but lower accuracy is achieved downstream. At $6 \mathrm{~mm}$ the PMD is over-predicted, however the spray width is well captured.

Two different inlet boundary condition were used in the RANS simulations: a mapped boundary condition, where all flow variables were taken from previous nozzle flow calculations [18], and a constant radially averaged outlet profile, which will be used to compare with LES cases.
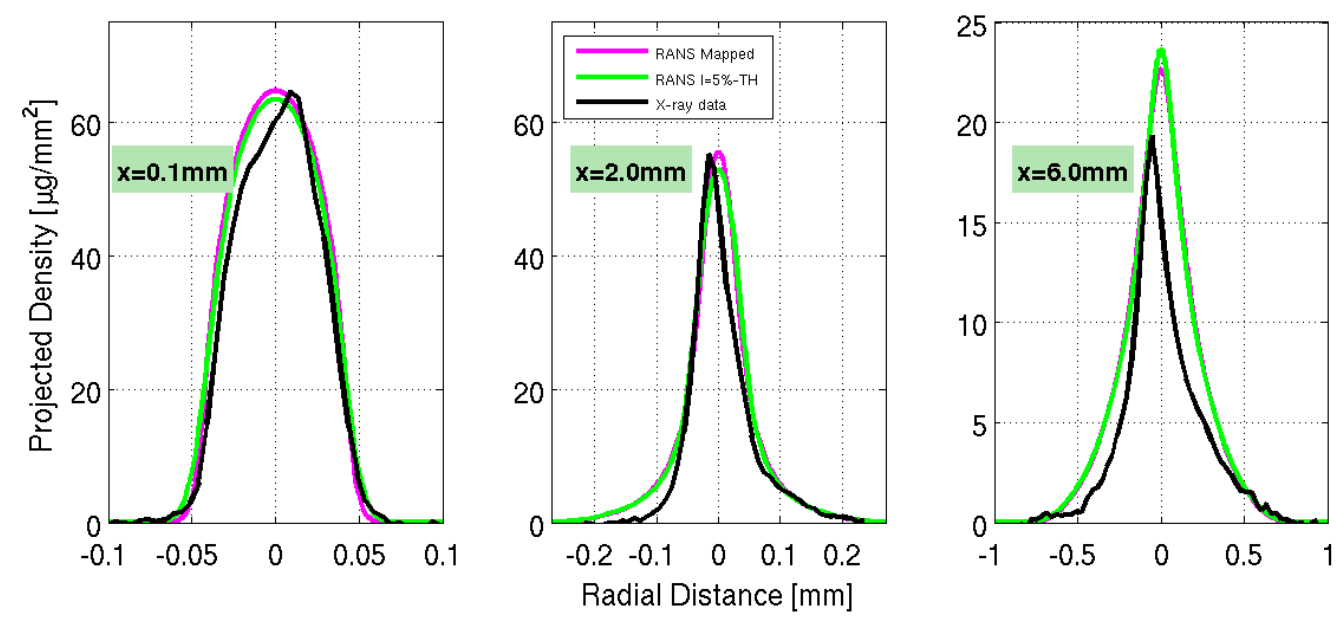

Figure 1. Measured and computed PMD at $0.1,2$ and $6 \mathrm{~mm}$ downstream the nozzle exit. $\mathrm{P}_{\text {inj }}=150 \mathrm{MPa}, \rho_{\mathrm{mmb}}=22.8 \mathrm{~kg} / \mathrm{m}^{3}$ 
LES simulations have been initially performed with a turbulent intensity of 5 -percent and $1 / 7^{\text {th }}$ power law mean velocity profile, as suggested in different LES Diesel spray simulations [11][35]. A single realization was run and time-averaging was performed from $0.5 \mathrm{~ms}$ after the Start of Injection, during the quasi-steady period, as in $\mathrm{x}$-ray experiments [24]. Figure 2 shows the instantaneous and averaged liquid mass fraction. The LES resolution is assessed by the ratio of SGS flow energy over total turbulent kinetic energy (modelled plus resolved). According to the criteria proposed in [36], this ratio (M) should be less than $20 \%$, which is accomplished in the simulations, as shown in RHS picture of Figure 2, within the region of interest for assessment.

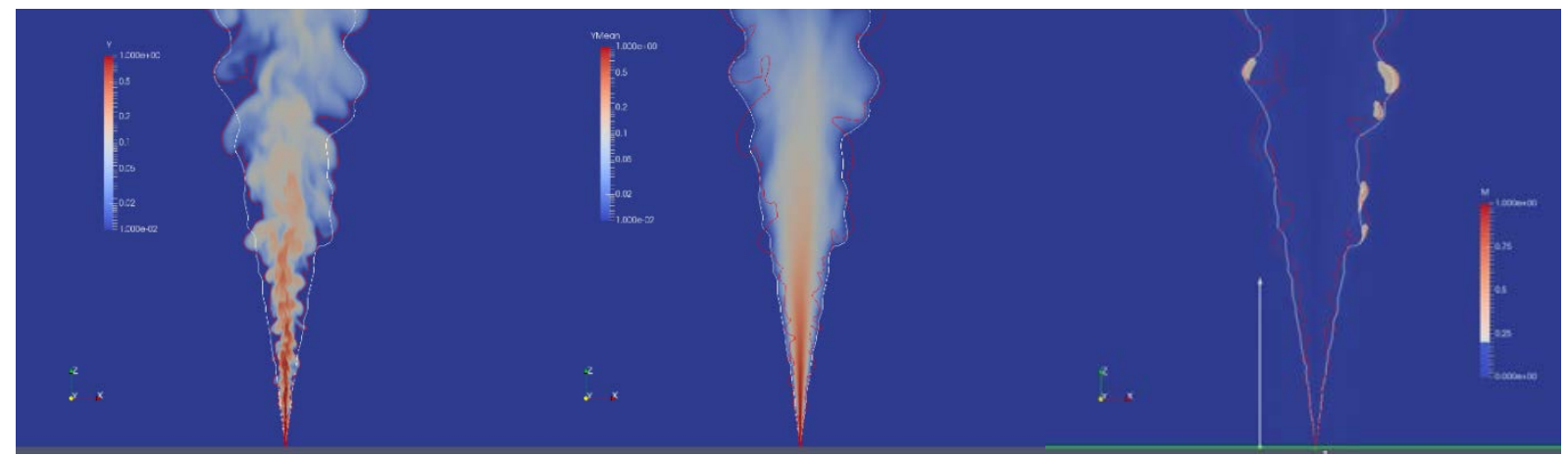

Figure 2. Cut-plane through spray axis showing instantaneous (left) and time-averaged (centre) liquid mass fraction. Modelledto-total flow kinetic energy ratio $M$ (right). Isolines of $1 \% \mathrm{Y}$ (white) and YMean (red). Arrow in right image is $15 \mathrm{~mm}$ length.

The PMD from time-averaged LES data are presented in Figure 3. This figure also includes results using the same mean velocity profile (top-hat) and turbulent intensity for both RANS and LES calculations. It is shown that LES captures PMD at the nozzle exit, but under-predicts at $2 \mathrm{~mm}$ downstream, and better results are obtained at $6 \mathrm{~mm}$, contrary to RANS, independently of the mean exit profile used (i.e. top-hat and power-law). This indicates that spray dispersion is over-predicted in the near-nozzle $(\sim 2 \mathrm{~mm})$ in LES calculations and the effect of the nozzle exit profile is reduced. Notice that the same mass and momentum flux is injected for both cases.

According to this result, the impact of fluctuations intensity was assessed in LES calculations. In this case a 3percent turbulence intensity, which corresponds to the outlet condition of a k- $\omega$ SST RANS nozzle flow simulation [19], has been used. Figure 4 shows a noticeable effect on PMD results when modifying initial fluctuations level, which have not been observed in previous RANS calculations [29]. It is shown that both 2 and $6 \mathrm{~mm}$ profiles predictions are improved with this lower fluctuation level boundary condition.
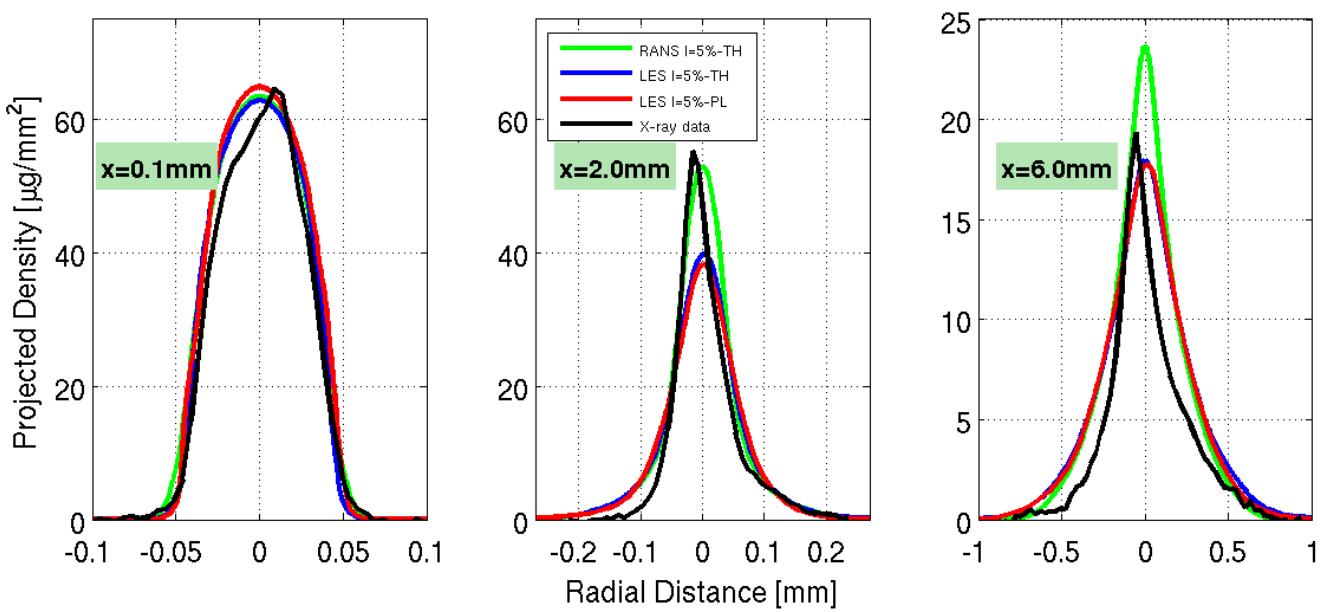

Figure 3. Measured and computed PMD at $0.1,2$ and $6 \mathrm{~mm}$ downstream the nozzle exit for RANS and LES simulations. Pinj $=$ $150 \mathrm{MPa}, \rho \mathrm{amb}=22.8 \mathrm{~kg} / \mathrm{m}^{3}$ 

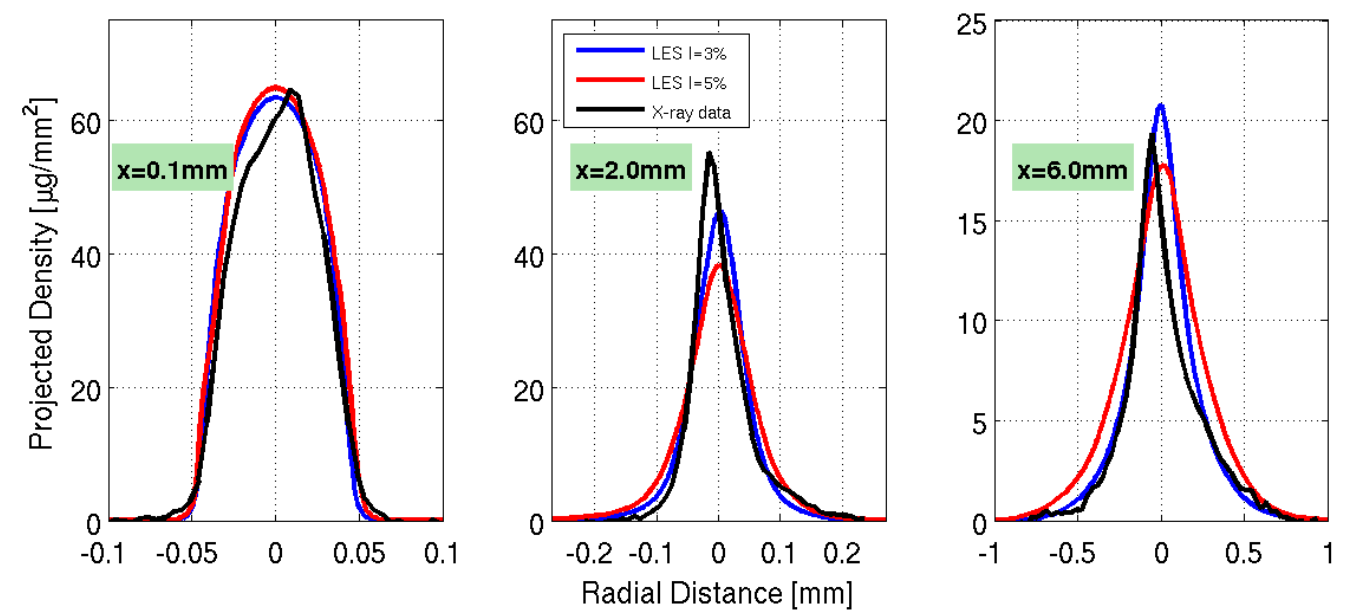

Figure 4. Measured and computed PMD at 0.1, 2 and $6 \mathrm{~mm}$ downstream the nozzle exit. Impact of turbulence intensity I in LES calculations. $P_{\text {inj }}=150 \mathrm{MPa}$, $\rho_{\mathrm{amb}}=22.8 \mathrm{~kg} / \mathrm{m}^{3}$

Figure 5 shows the Transverse Integrated Mass (TIM) which is obtained from the integral of the PMD across the transverse position for each axial location [1]. TIM is related to spray dispersion, so higher TIM indicates faster mixing [18]. It is shown that RANS modelling tends to increasingly over-predict TIM from the nozzle exit and thus spray mixing, while LES calculations are closer to experimental data up to $6 \mathrm{~mm}$. Downstream of this position TIM is over-predicted for both turbulence modelling approaches and also other works [4], but LES modelling has the potential to improve the predictions using lower turbulence intensity boundary conditions.

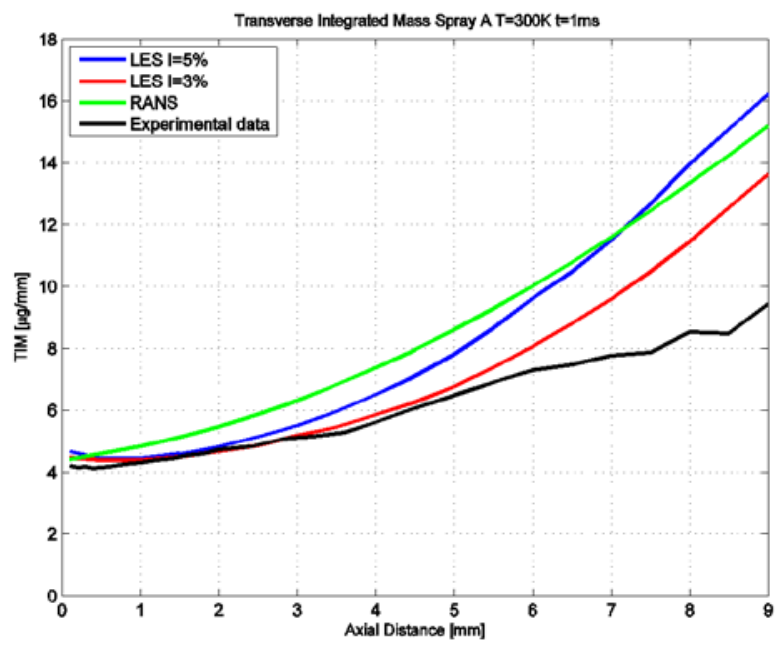

Figure 5. Measured and computed TIM along the spray axis RANS and LES simulations. $\operatorname{Pinj}=150 \mathrm{MPa}, \rho_{\mathrm{mmb}}=22.8 \mathrm{~kg} / \mathrm{m}^{3}$

\section{Spray atomization: Surface density results}

As explained in the modelling approach section, the two modelling constants of the surface density equation should be calibrated. The expertise acquired recently [23] suggests the capital importance of the $\alpha_{2}$ constant while $\alpha_{1}$ effects (with a value in the vicinity of 1.0) could be negligible. Thus, the fist constant has been set to unity and the calibration of $\alpha_{2}$ parameter has been made. The starting point is an evaluation of the results achieved with the $\alpha_{1}$ values obtained for limiting conditions of the critical Weber number from [35] $\left(\mathrm{We}_{\mathrm{c}} \sim 6\right.$ 6-15), see Table 2:

Table 2. Reference studies conducted for the interphase surface density equation setup

\begin{tabular}{|c|c|c|}
\hline Case & $\boldsymbol{\alpha}_{1}[-]$ & $\boldsymbol{\alpha}_{2}[-]$ \\
\hline Reference 1 & 1.0 & 0.16 \\
\hline Reference 2 & 1.0 & 0.06 \\
\hline
\end{tabular}


In Figure 6, the results for the two reference cases evaluated are plotted on the left. It is shown both, the CFD surface area (solid line) and the equilibrium value (pointed line) together with the experimental measurements (black dashed line). These configurations over-predict the surface area results and it is clear that a lower $\alpha_{2}$ value is needed to be able to match these measurements. Then, four more simulations are conducted with the following values for the second parameter: $0.04,0.035,0.03$ and 0.02 , these new predictions are depicted in Figure 6 , on the right. It can be seen the great scalability achieved for the surface density predictions as well as the most suitable $\alpha_{2}$ constant value. According to the results, the experimental axial profile is almost completely matched with a parameter value of 0.035 . As a result, it is chosen as the optimum for the following calculations.
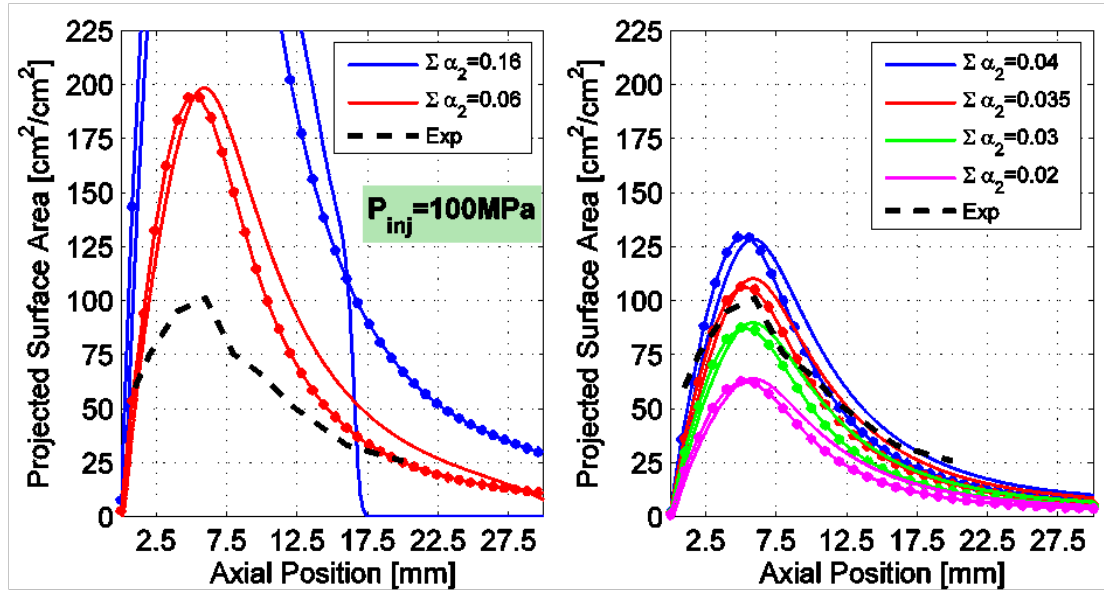

Figure 6. Projected surface area results at $1.5 \mathrm{~ms}$ after SOI for different values of the second modelling constant. $P_{\mathrm{inj}}=100$ $\mathrm{MPa}, \rho_{\mathrm{amb}}=22.8 \mathrm{~kg} / \mathrm{m} 3$

Finally, the optimum chosen setup is evaluated at the different operating conditions available. These results are depicted again at $1.5 \mathrm{~ms}$ after SOI in Figure 7 . The influence of injection pressure is shown on the left while the back pressure effect, i.e. ambient density, is shown on the right. Experimental trends are well reproduced; decreased injection pressure decreases the surface area profile, as well as the location at which the maximum occurs. However, a little deviation from the experimental data is noticeable for high and low injection pressure conditions. On the other hand, quite remarkable performance is achieved for low ambient density case, which clearly improves recent predictions [23].


Figure 7. Projected surface area results at $1.5 \mathrm{~ms}$ after SOI for optimum set-up. The computational predictions are the continuous lines and the experimental measurements are the dashed lines.

\section{Conclusions}

This paper presents an assessment of the $\Sigma-Y$ model in the near-nozzle region of the ECN spray A. Unique experimental techniques performed at Argonne NL, namely X-ray radiography and USAXS, have been used to perform a direct evaluation of the model predictions. 
It is shown that the model is able to capture liquid spray dispersion in the near nozzle region, using previous RANS and newly performed LES calculations. Spray dispersion predictions accuracy can be improved by LES modelling, thought noticeably sensitivity to inflow turbulence intensity levels has been observed, which requires further insight. The interfacial surface evolution model has been directly compared to measurements obtained using USAXS technique. A new formulation for critical surface density has been used and the model was calibrated in a single operation point. Without further adjustment the model was able to fairly predict injection pressure variations, and especially lower ambient density condition, improving previous formulation results.

In order to perform further model evaluation and confirm predictive capabilities, different operating conditions and injectors must be considered.

\section{Acknowledgements}

Authors acknowledge that part of this work was partially funded by the Spanish Ministry of Economy and Competitiveness in the frame of the COMEFF (TRA2014-59483-R) project.

Parts of this research were performed at the 7-BM and 9-ID beam lines of the Advanced Photon Source at Argonne National Laboratory. Use of the APS is supported by the U.S. Department of Energy (DOE) under Contract No. DEAC02-06CH11357. The research was partially funded by DOE's Vehicle Technologies Program, Office of Energy Efficiency and Renewable Energy.

The authors would like to thank Team Leaders Gurpreet Singh and Leo Breton for their support of this work

\section{References}

[1] Kastengren, A.L., Powell, C.F., Wang, Y., Im, K.-S. et al., 2009, Atomization and. Sprays 19 (11), pp. 10311044.

[2] Linne, M., Paciaroni, M., Hall, T. et al., 2006, Exp. Fluids, 40, pp. 836-846.

[3] Dukowicz, J.K., 1980, Journal of Computational Physics 35 (2), pp.229-253.

[4] Xue, Q., Battistoni, M., Powell, C. F., Longman, D. E. et al., 2015, Int. Jou.Multiph. Flow 70 pp. 77-88.

[5] Desantes, J.M., Garcia-Oliver, J.M., Pastor, J.M. and Pandal, A., 2016, Atomization and Sprays 26 (7)

[6] Gorokhovski, M. and Herrmann, M., 2008, Annu. Rev. Fluid Mech. 40 pp. 343-366.

[7] Lebas, R., Menard, T., Beau, P.-A., Berlemont, A. et al., 2009, Int. Jou. Multiph. Flow, 35(3) pp. 247-260.

[8] Arienti, M. and Sussman, M., 2017, Int. Jou. Multiph. Flow 88, pp. 205-221.

[9] Vallet, A. and Borghi, R., 1999, C.R. Acad. Sci, Paris, 327, pp. 1015-1020.

[10] Vallet, A., Burluka, A. and Borghi, R., 2001, Atomization and Sprays 11, pp. 619-642

[11] Lacaze, G., Misdariis, A., Ruiz, A., Oefelein, J. C., 2015, Proceedings of the Combustion Institute 35 (2) pp. 1603-1611.

[12] Blokkeel, G., Barbeau, B., Borghi, R., 2003, SAE Technical Paper 2003-01-005.

[13] Lebas R., Blokkeel, G, Beau, P.A., Demoulin, F.-X., Aug 1-Sep 27, 2006, ICLASS.

[14] Demoulin, F.-X., Beau, P. A., Blokkeel, G., Mura, A. et al., 2007, Atomization and Sprays 17(4).

[15] Demoulin, F.-X., Reveillon, J., Duret, B., Bouali, Z.et al., 2013, Atomization and Sprays 23 (11).

[16] Engine Combustion Network, https://ecn.sandia.gov/ (cit. 2016-12-20).

[17] Kastengren, A., llavsky, J., Viera, J., Payri, R. et al., 2017, Int. J. Multiph. Flow (In Press).

[18] Desantes, J., García-Oliver, J., Pastor, J., Pandal, A. et al., 2016, Int. J. Multiph. Flow 80, pp. 89-99.

[19] Salvador, F.J., Gimeno, J, Pastor, J.M., Martí-Aldaraví, P., 2014, Int. J. Multiph. Flow 65, pp. 108-116.

[20] Garcia-Oliver, J.M., Pastor, J.M., Pandal, A., Trask, N. et al.., 2013, Atomization and Sprays 23 (1)

[21] Beheshti, N., Burluka, A., Fairweather, M., 2007, Theoretical and Computational Fluid Dynamics 21 pp. $381-$ 397.

[22] Duret B., Reveillon, J., Menard, T., Demoulin, F.-X, 2013, Int. J. Multiph. Flow 55, pp. 130-137.

[23] Pandal, A., Pastor, J.M, Payri, R., Kastengren et al., 2017, SAE Int. J. Fuels Lubr. 10(2)

[24] Kastengren A.L., Tilocco F.Z., Duke D., Powell C.F. et al., Sep 2-6, 2012, $12^{\text {th }}$ Triennial International Conference on Liquid Atomization and Spray Systems.

[25] Ilavsky, J., Jemian, P. R., Allen, A. J., Zhang, F. et al., J. Appl. Cryst. 42(3), pp. 469-479.

[26] Ilavsky, J. and Jemian, P. R., J. Appl. Cryst. 42(2):347-353.

[27] Naber, J. and Siebers, D., 1996, SAE Technical Paper 960034.

[28] Weller, H. G., Tabor, G., Jasak, H., Fureby, C., 1998, Computers in Physics, 12(6),pp. 620-631.

[29] Pandal, A., Pastor, J.M, García-Oliver, J.M., Schmidt, D.P., 2016, Int. J. Multiph. Flow 83, pp. $162-171$.

[30] Kraichan R., 1970, Phys. Fluids 13, pp. 22-31.

[31] Klein M., Sadiki A., Janicka J., 2003, J. Comput. Phys.186, pp. 652-665.

[32] Robert A., Martinez, L., Tillou, J., Richard, S., 2014, Oil \&Gas Science and Technology 69, pp.141-1551.

[33] Pope, S., 1978, AIAA 16, pp. 279-281.

[34] Nicoud, F, Baya-Toda, H., Cabrit, O., Bose, S. et al., 2011, Physic of Fluids, 23, 085106.

[35] Chesnel, J., Reveillon, J., Menard, T., Demoulin, F.-X, 2011, Atomization and Sprays, 21(9), pp. 711-736.

[36] Pope,S.B., 2004, New J. Phys. 6, 35. 